\title{
CO (2-1) LINE EMISSION IN REDSHIFT 6 QUASAR HOST GALAXIES
}

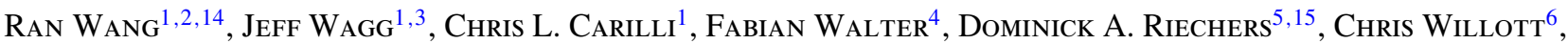 \\ Frank Bertoldi $^{7}$, Alain Omont $^{8}$, Alexandre Beelen $^{9},{\text { Pierre } \text { Cox }^{10} \text {, Michael A. Strauss }}^{11}$, \\ Jacqueline Bergeron $^{8}$, Thierry Forveille ${ }^{12}$, Karl M. Menten ${ }^{13}$, and Xiaohui Fan ${ }^{2}$ \\ ${ }^{1}$ National Radio Astronomy Observatory, P.O. Box 0, Socorro, NM 87801, USA \\ ${ }^{2}$ Steward Observatory, University of Arizona, 933 N Cherry Ave., Tucson, AZ 85721, USA \\ ${ }^{3}$ European Southern Observatory, Alonso de Córdova 3107, Vitacura, Casilla 19001, Santiago 19, Chile \\ ${ }^{4}$ Max-Planck-Institute for Astronomy, Königsstuhl 17, 69117 Heidelberg, Germany \\ ${ }^{5}$ California Institute of Technology, 1200 E. California Blvd., Pasadena, CA 91125, USA \\ ${ }^{6}$ Herzberg Institute of Astrophysics, National Research Council, 5071 West Saanich Rd., Victoria, BC V9E 2E7, Canada \\ ${ }^{7}$ Argelander-Institut für Astronomie, University of Bonn, Auf dem Hügel 71, 53121 Bonn, Germany \\ ${ }^{8}$ Institut d'Astrophysique de Paris, CNRS and Université Pierre et Marie Curie, Paris, France \\ ${ }^{9}$ Institut d'Astrophysique Spatiale, Université Paris-Sud, F-91405 Orsay Cedex, France \\ ${ }^{10}$ Institute de Radioastronomie Millimetrique, St. Martin d'Heres, F-38406, France \\ ${ }^{11}$ Department of Astrophysical Sciences, Princeton University, Princeton, NJ 08544, USA \\ ${ }^{12}$ Laboratoire d'Astrophysique, Observatoire de Grenoble, Université J. Fourier, BP 53, F-38041 Grenoble, Cedex 9, France \\ ${ }^{13}$ Max-Planck-Institut für Radioastronomie, Auf dem Hügel 69, 53121 Bonn, Germany \\ Received 2011 April 15; accepted 2011 May 16; published 2011 August 29
}

\begin{abstract}
We report new observations of $\mathrm{CO}(2-1)$ line emission toward five $z \sim 6$ quasars using the $K a$-band receiver system on the Expanded Very Large Array (EVLA). Strong detections were obtained in two of them, SDSS J092721.82+200123.7 and CFHQS J142952.17+544717.6, and a marginal detection was obtained in another source, SDSS J084035.09+562419.9. Upper limits of the CO (2-1) line emission have been obtained for the other two objects. The CO (2-1) line detection in J0927+2001 together with previous measurements of the CO (6-5) and (5-4) lines reveal important constraints on the $\mathrm{CO}$ excitation in the central $\sim 10 \mathrm{kpc}$ region of the quasar host galaxy. The CO (2-1) line emission from J1429+5447 is resolved into two distinct peaks separated by $1^{\prime \prime} .2(\sim 6.9 \mathrm{kpc})$, indicating a possible gas-rich, major merging system, and the optical quasar position is consistent with the west peak. This result is in good agreement with the picture in which intense host galaxy star formation is coeval with rapid supermassive black hole (SMBH) accretion in the most distant universe. The two EVLA detections are ideal targets for further high-resolution imaging (e.g., with ALMA or EVLA observations) to study the gas distribution, dynamics, and SMBH-bulge-mass relation in these earliest quasar host galaxy systems.
\end{abstract}

Key words: galaxies: active - galaxies: evolution - galaxies: high-redshift - molecular data - radio lines: galaxies

Online-only material: color figure

\section{INTRODUCTION}

Observations of high-redshift quasars probe the growth of supermassive black holes and their connection to galaxy formation at the earliest cosmic epochs. The discovery of strong submillimeter/millimeter ((sub)mm) dust continuum in about $30 \%$ of the quasars known at $z \sim 6$ provides the first evidence of active star formation in young quasar host galaxies at the end of the reionization era (Bertoldi et al. 2003a, 2003b; Petric et al. 2003; Priddey et al. 2003; Robson et al. 2004; Wang et al. 2007, 2008). The star formation rates estimated from the far-IR (FIR) luminosities (a few $10^{12}$ to $10^{13} L_{\odot}$ ) are on the order of $10^{2}$ to $10^{3} M_{\odot} \mathrm{yr}^{-1}$, which are comparable to the typical values found in the so-called submillimeter galaxies at $z=2 \sim 3$ (Scott et al. 2002; Greve et al. 2005; Kovács et al. 2006). The spatially resolved [C $\mathrm{II}]$ line emission from one of the most FIR luminous $z \sim 6$ quasars, SDSS J114816.64+525150.3 (hereafter $\mathrm{J} 1148+5251$ ), further suggests a high star formation surface density of $\sim 1000 M_{\odot} \mathrm{yr}^{-1} \mathrm{kpc}^{-2}$ over the central $1.5 \mathrm{kpc}$ region of the quasar host galaxy (Maiolino et al. 2005; Walter et al. 2009).

Molecular CO (6-5) line emission has been detected in 10 of the FIR luminous $z \sim 6$ quasars (Bertoldi et al. 2003b; Walter

\footnotetext{
${ }_{14}^{14}$ Jansky Fellow.

${ }^{15}$ Hubble Fellow.
}

et al. 2003; Carilli et al. 2007; Wang et al. 2010; R. Wang et al. 2011 , in preparation), indicating the existence of highly excited molecular gas in the quasar hosts. The CO (3-2), (6-5), and (7-6) transitions detected in the $z=6.42$ quasar J1148+5251 reveal a molecular gas component on scales of $\sim 5 \mathrm{kpc}$ in the host galaxy with $\mathrm{CO}$ excitation conditions similar to those found in local starburst galaxies and CO-detected quasars at lower redshifts (Bertoldi et al. 2003b; Walter et al. 2004; Riechers et al. 2009).

Emission in the low-order $\mathrm{CO}$ transitions $(J \leqslant 2)$ from the $z \sim 6$ quasar host galaxies is poorly constrained due to the limited sensitivity and frequency coverage of the previous instruments (Wagg et al. 2008; Wang et al. 2010). The new $K a$-band receivers on the Expanded Very Large Array (EVLA) open an important frequency window for studies of the cold molecular gas in high-redshift galaxies (e.g., Ivison et al. 2010, 2011; Riechers et al. 2010). In this Letter, we report our EVLA observations of the CO (2-1) line emission in five $z \sim 6$ quasars (Fan et al. 2004, 2006; Willott et al. 2010a, 2010b). Three of them are from the Sloan Digital Sky Survey (SDSS; Fan et al. 2004, 2006), with two objects, SDSS J084035.09+562419.9 and SDSS J092721.82+200123.7, previously detected in strong $(>3 \mathrm{mJy}) 250 \mathrm{GHz}$ dust continuum and molecular CO (6-5) and (5-4) line emission. Another object, SDSS J162331.81+311200.5, was detected in the [C II] 
Table 1

Observations

\begin{tabular}{|c|c|c|c|c|c|c|c|}
\hline Name & $\begin{array}{l}\mathrm{t}_{\mathrm{obs}} \\
(\mathrm{hr}) \\
(2)\end{array}$ & Configuration & $\begin{array}{l}\text { Redshift } \\
\text { (4) }\end{array}$ & $\begin{array}{c}\text { FWHM } \\
\left(\mathrm{km} \mathrm{s}^{-1}\right) \\
(5)\end{array}$ & $\begin{array}{c}I \Delta v \\
\left(\mathrm{Jy} \mathrm{km} \mathrm{s}^{-1}\right) \\
(6)\end{array}$ & $\begin{array}{c}L_{\mathrm{CO}(2-1)}^{\prime} \\
\left(10^{10} \mathrm{~K} \mathrm{~km} \mathrm{~s}^{-1} \mathrm{pc}^{2}\right) \\
(7)\end{array}$ & $\begin{array}{c}M_{\text {gas }} \\
\left(10^{10} M_{\odot}\right) \\
(8)\end{array}$ \\
\hline SDSS J092721.82+200123.7 & 20 & $\mathrm{D}, \mathrm{DnC}$ & $5.7716 \pm 0.0012$ & $590 \pm 130$ & $\begin{array}{l}0.129 \pm 0.018^{\mathrm{a}} \\
0.202 \pm 0.040^{\mathrm{b}}\end{array}$ & $\begin{array}{l}3.70 \pm 0.52^{\mathrm{a}} \\
5.80 \pm 1.15^{\mathrm{b}}\end{array}$ & $\begin{array}{l}3.0 \pm 0.4^{\mathrm{a}} \\
4.6 \pm 0.9^{\mathrm{b}}\end{array}$ \\
\hline CFHQS J142952.17+544717.6W & 15 & $\mathrm{C}$ & $6.1831 \pm 0.0007$ & $280 \pm 70$ & $0.065 \pm 0.011$ & $2.06 \pm 0.35$ & $1.6 \pm 0.3$ \\
\hline CFHQS J142952.17+544717.6E & 15 & $\mathrm{C}$ & $6.1837 \pm 0.0015$ & $400 \pm 140$ & $0.050 \pm 0.013$ & $1.59 \pm 0.41$ & $1.3 \pm 0.3$ \\
\hline SDSS J084035.09+562419.9 & 20 & $\mathrm{D}, \mathrm{DnC}, \mathrm{C}$ & $5.8441^{\mathrm{d}}$ & $860^{\mathrm{d}}$ & $0.062 \pm 0.022$ & $1.81 \pm 0.64$ & $1.4 \pm 0.5$ \\
\hline CFHQS J021013.19-045620.9 & 15 & $\mathrm{C}$ & $6.438^{\mathrm{e}}$ & $\ldots$ & $<0.038$ & $<1.28$ & $<1.0$ \\
\hline SDSS J162331.81+311200.5 & 20 & $\mathrm{D}$ & $6.26^{\mathrm{f}}$ & $\cdots$ & $<0.062$ & $<2.00$ & $<1.6$ \\
\hline
\end{tabular}

Notes.

${ }^{\text {a }} \mathrm{CO}(2-1)$ line flux and luminosity derived from the peak surface brightness on the velocity-averaged map.

${ }^{\mathrm{b}} \mathrm{CO}(2-1)$ line flux and luminosity derived from the total intensity integrated over the line-emitting area on the velocity-averaged map.

${ }^{c}$ The west component of J1429+5447 is consistent with the optical quasar position.

d Redshift and CO line width measured from the CO (6-5) and (5-4) lines (Wang et al. 2010).

e Redshift measured from the quasar Mg II line emission (Willott et al. 2010b).

${ }^{\mathrm{f}}$ Redshift from the C II line detection (F. Bertoldi et al. 2011, in preparation)

line, but undetected in millimeter dust continuum and high$J$ CO transitions (F. Bertoldi et al. 2011, in preparation). The other two objects are from the Canada-France High$z$ Quasar Survey (CFHQS; Willott et al. 2010a, 2010b) and do not have published $\mathrm{CO}$ observations yet. One of them, CFHQS 142952.17+544717.6, was detected in $250 \mathrm{GHz}$ dust continuum (A. Omont et al. 2011, in preparation). We describe the observations in Section 2, present the results in Section 3, and discuss the $\mathrm{CO}$ excitation and host galaxy evolution properties of the detections in Section 4. A $\Lambda$-CDM cosmology with $\mathrm{H}_{0}=71 \mathrm{~km} \mathrm{~s}^{-1} \mathrm{Mpc}^{-1}, \Omega_{\mathrm{M}}=0.27$, and $\Omega_{\Lambda}=0.73$ is adopted throughout this Letter (Spergel et al. 2007).

\section{OBSERVATIONS}

The observations were carried out using the $K a$-band receiver on the EVLA (Perley et al. 2011) in 2010 in the D, DnC, and $\mathrm{C}$ configurations. The WIDAR correlator in Open Shared Risk Observing mode provided a maximum bandwidth of $128 \mathrm{MHz}$ and a resolution of $2 \mathrm{MHz}$ in each of the two basebands (A/C and B/D intermediate frequency (IF) bands). The A/ $\mathrm{C}$ IFs could not be tuned below $32 \mathrm{GHz}$. The redshifts and observing frequencies of the $\mathrm{CO}(2-1)$ line of the five targets are estimated with previous detections of the $\mathrm{CO}(6-5),[\mathrm{C} \mathrm{II}]$, or quasar UV lines (Carilli et al. 2007; Wang et al. 2010; F. Bertoldi et al. 2011, in preparation). For the three sources with redshifts of $z \leqslant 6.2$ (corresponding to redshifted CO (2-1) line frequencies of $v_{\mathrm{obs}} \geqslant 32 \mathrm{GHz}$ ), we use the two $128 \mathrm{MHz} \mathrm{IF}$ pairs overlapped by $30 \mathrm{MHz}$ and cover a total bandwidth of $226 \mathrm{MHz}$ (i.e., $\sim 2000 \mathrm{~km} \mathrm{~s}^{-1}$ in velocity and $\sim 0.05$ in redshift at $z=6) .{ }^{16}$ For the other two objects with $z>6.2$, we centered the $128 \mathrm{MHz}$ window of the B/D IF pairs on the line frequency and observed the continuum at $\geqslant 32 \mathrm{GHz}$ with the other window. The observing time is $15-20 \mathrm{hr}$ for each of the five targets (see Table 1). Flux calibrations were performed using the standard VLA calibrators, 3C286 and 3C48, and we use five-minute scan loops between targets and phase calibrators to calibrate the phase. The data were reduced with AIPS, and the spatial resolutions (FWHM) of the final images are typically $2^{\prime \prime}$ for data taken in the $\mathrm{D}$ configuration and 0.7 for the $\mathrm{C}$ configuration.

\footnotetext{
$1610 \mathrm{MHz}$ overlap and a total bandwidth of $246 \mathrm{MHz}$ for J1429+5447.
}

\section{RESULT}

CO (2-1) line emission has been detected in two of the five $z \sim 6$ quasars, J0927+2001 and J1429+5447, and marginally detected in $\mathrm{J} 0840+5624$. We present all the observing parameters and measurements in Table 1 . The detailed results are listed below.

$J 0927+2001$. Toward this source strong dust continua at $850 \mathrm{GHz}, 250 \mathrm{GHz}$, and $85 \mathrm{GHz}$, and $\mathrm{CO}(6-5)$ and (5-4) line emission were detected (Carilli et al. 2007; Wang et al. 2010). We have detected the CO (2-1) line, and the emission distribution (averaged over a velocity range of $880 \mathrm{~km} \mathrm{~s}^{-1}$ ) along with a spectrum is shown in Figure 1. The line peak emission centroid is consistent with the optical quasar position and the peaks of the high- $J$ CO lines. The line width (FWHM) and redshift fitted with a single Gaussian profile are $590 \pm$ $130 \mathrm{~km} \mathrm{~s}^{-1}$ and $5.7716 \pm 0.0012$ which are in good agreement with the measurements from the high-order $\mathrm{CO}$ transitions $\left(z=5.7722 \pm 0.0006\right.$ and FWHM $=600 \pm 70 \mathrm{~km} \mathrm{~s}^{-1}$; Carilli et al. 2007). The line emission appears marginally resolved by the 2 .' $19 \times 1$ ".96 synthesized beam with a peak surface brightness of $147 \pm 21 \mu \mathrm{Jy} \mathrm{beam}^{-1}$ and a total intensity of $230 \pm 45 \mu \mathrm{Jy}$, with a source size of $\left(22^{\prime \prime} 7 \pm 0.4\right) \times(2.4 \pm 0.3)$ determined from a fit with a two-dimensional Gaussian distribution (the deconvolved source size is about $1^{\prime \prime} .7 \times 1^{\prime \prime}$. 4 , or $10 \mathrm{kpc} \times 8 \mathrm{kpc}$ ). The corresponding line fluxes and luminosities (Table 1) are higher than the upper limits estimated from previous Green Bank Telescope (GBT) observations, but are still consistent given the large uncertainties and baseline feature contamination in the GBT data (Wagg et al. 2008; Wang et al. 2010).

$J 1429+5447$. Toward this object strong radio continuum emission was detected in the FIRST survey (Becker et al. 1995) and in recent very long baseline interferometry observations (Frey et al. 2011), making it the strongest radio source among the known $z \sim 6$ quasars and the most distant radio-loud quasar. It has also been detected in dust continuum at $250 \mathrm{GHz}$ with a flux density of $\sim 3 \mathrm{mJy}$ (A. Omont et al. 2011, in preparation). We have detected both $\mathrm{CO}(2-1)$ line emission and continuum emission at the line frequency. The continuum source is unresolved by the $00^{\prime} .71 \times 00^{\prime} .67$ synthesized beam and the flux density averaged over the line-free channels at $32 \mathrm{GHz}$ is $257 \pm 15 \mu \mathrm{Jy}$. We subtract the continuum by performing linear fitting to the visibility data, using the 

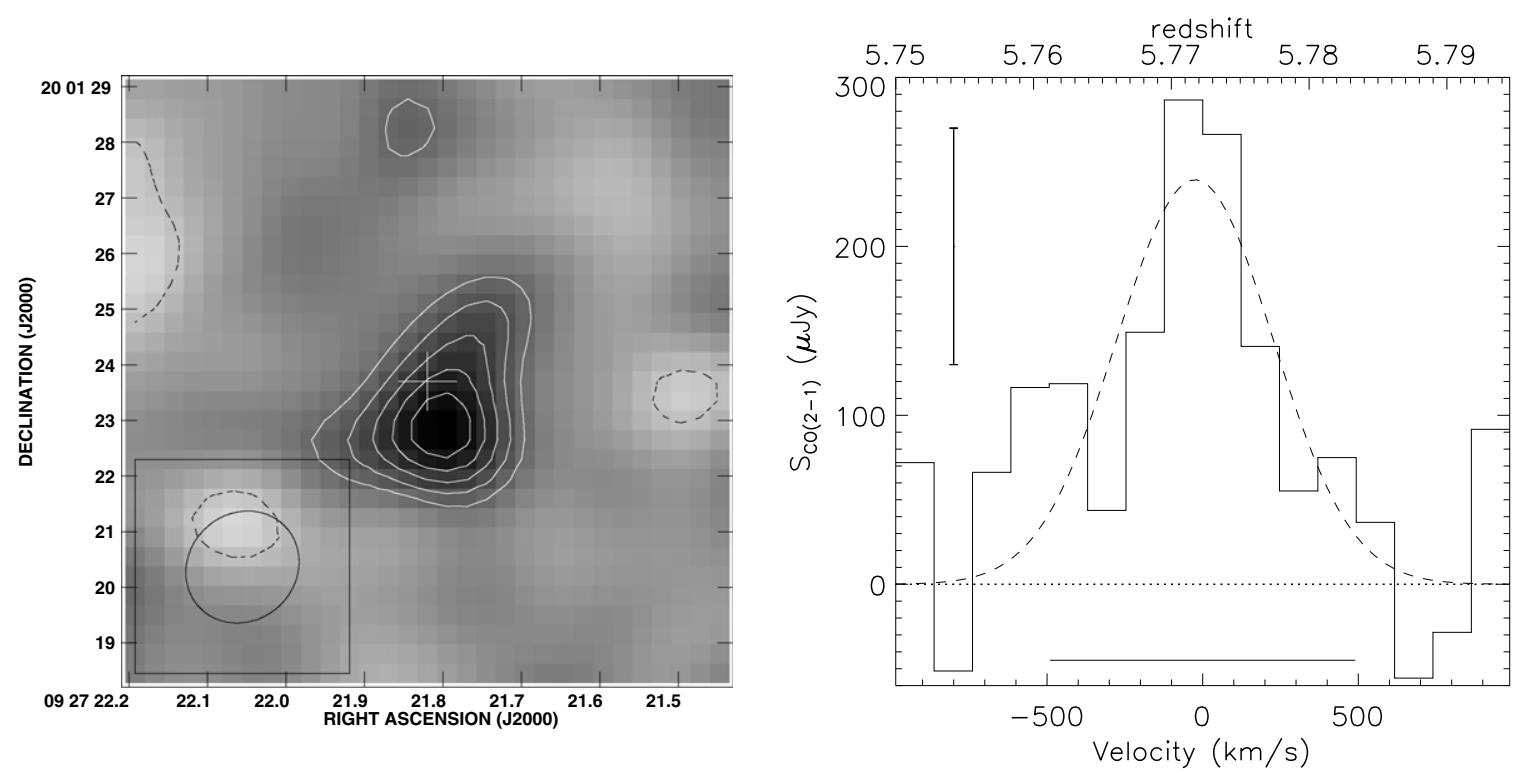

Figure 1. Left: velocity-averaged map of the CO (2-1) line emission from J0927+2001 at a resolution of $2^{\prime \prime} .19 \times 1$ 1".96, averaged over $\sim-440$ to $440 \mathrm{~km} \mathrm{~s}{ }^{-1}$. The contour levels are $(-2,2,3,4,5) \times 21 \mu \mathrm{Jy}_{\text {beam }}^{-1}$. The cross denotes the optical quasar position. The source appears marginally resolved with a source size of 2 '.7 $\times 2$ 2.4 fit to a two-dimensional Gaussian profile (deconvolved size of $1^{\prime \prime} 7 \times 1$ 1". 4 , or $10 \mathrm{kpc} \times 8 \mathrm{kpc}$ ). Right: spectrum integrated over $\sim 2$ beam area. The spectrum is binned to a channel width of $14 \mathrm{MHz}\left(123 \mathrm{~km} \mathrm{~s}^{-1}\right)$ and the zero velocity corresponds to the high- $J$ CO redshift of $z=5.7722$ (Carilli et al. 2007). The error bar represents the typical $\pm 1 \sigma \mathrm{rms}$ in each channel. The dashed line denotes a Gaussian fit to the line and the dotted line shows the zero intensity level. The solid line at the bottom shows $v_{\text {FWZI }}$ of the high- $J$ CO lines (Carilli et al. 2007).
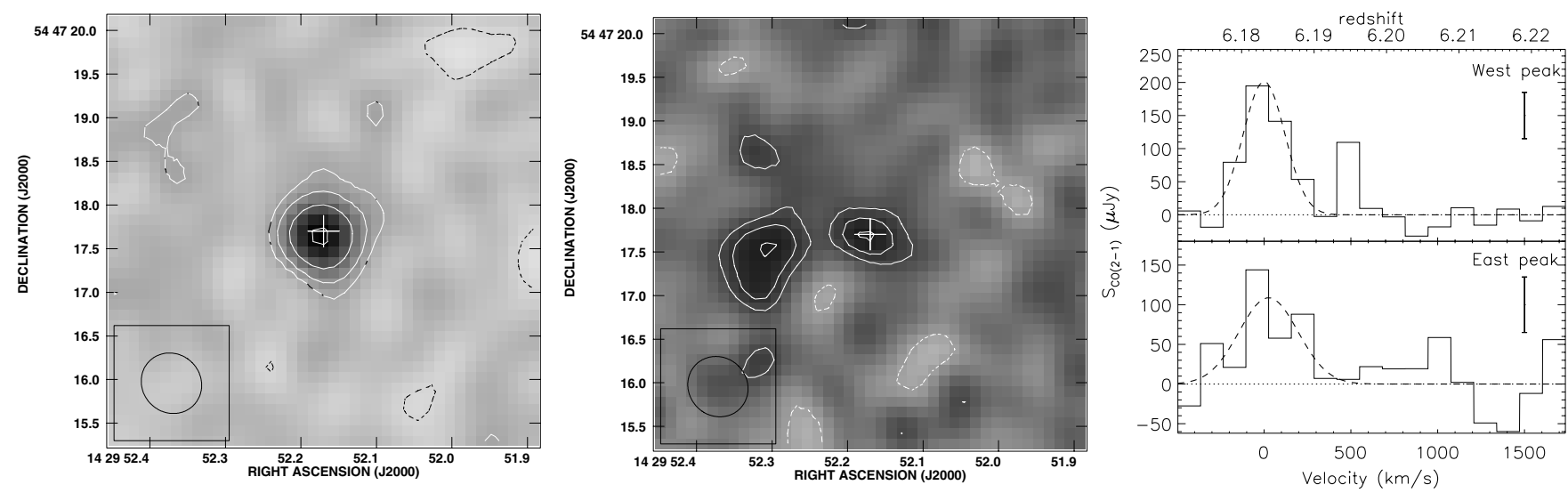

Figure 2. Left: $32 \mathrm{GHz}$ continuum emission from $\mathrm{J} 1429+5447$ at a resolution of $0^{\prime \prime} \cdot 71 \times 00^{\prime \prime} 67$. The contour levels are $(-2,2,4,8,16) \times 15 \mu \mathrm{Jy}$ beam ${ }^{-1}$. The cross denotes the optical position of the quasar. Middle: velocity-averaged map of the $\mathrm{CO}(2-1)$ line emission, averaged from $\sim-100$ to $340 \mathrm{~km} \mathrm{~s}{ }^{-1}$ to get the best signal-to-noise for both components. The contour levels are $(-2,2,3,4) \times 27 \mu \mathrm{Jy}$ beam ${ }^{-1}$. The source is resolved into two peaks with a separation of $\sim 1^{\prime \prime} \cdot 2(6.9 \mathrm{kpc})$, and the quasar position is consistent with the west peak. Right: spectra of the two components on the map. The quasar continuum has been subtracted from the west component. We adopt the surface brightnesses at the peak positions as the measurements of the line flux densities in each channel. The spectra are binned to a channel width of $14 \mathrm{MHz}\left(130 \mathrm{~km} \mathrm{~s}^{-1}\right)$ and the zero velocity corresponds to the redshift of $z=6.1831$ measured with the west component. The dashed lines denote a Gaussian fit and the dotted lines show the zero intensity level. The spike at the 8th channel (i.e., $\sim 500 \mathrm{~km} \mathrm{~s}^{-1}$ ) of the upper panel is due to the poor sensitivity ( $\sigma \sigma \mathrm{rms}$ of $\sim 160 \mu \mathrm{Jy}_{\text {beam }}^{-1}$ ) at the edge of the A/C IFs. The error bar represents the typical $\pm 1 \sigma \mathrm{rms}$ in the rest channels.

UVLIN task in AIPS. The CO line emission is resolved into two peaks with a spatial separation of $\sim 1^{\prime \prime} .2(6.9 \mathrm{kpc}$ at the quasar redshift), and the optical and radio quasar positions are consistent with the west peak (Figure 2). A Gaussian fit to the spectra yields a redshift of $z=6.1831 \pm 0.0007$ and a line width of FWHM $=280 \pm 70 \mathrm{~km} \mathrm{~s}^{-1}$ for the west source, and $z=6.1837 \pm 0.0015$ and FWHM $=400 \pm 140 \mathrm{~km} \mathrm{~s}^{-1}$ for the east source. The line fluxes estimated with the peak surface brightness on the velocity-averaged map averaging over a velocity range of $\sim 450 \mathrm{~km} \mathrm{~s}^{-1}$ are $0.065 \pm 0.011 \mathrm{Jy} \mathrm{km} \mathrm{s}^{-1}$ and $0.050 \pm 0.013 \mathrm{Jy} \mathrm{km} \mathrm{s}^{-1}$ for the west and east components, respectively. However, a two-dimensional Gaussian distribution fitted to the east component suggests possible extension with a source size of $\left(1^{\prime \prime} 1 \pm 00^{\prime} 2\right) \times\left(0^{\prime} 7 \pm 0 ! 2\right)$, which should be checked with deeper observations at higher spatial resolution.

$J 0840+5624$. This source was detected in (sub)mm dust continuum emission and CO (6-5) and (5-4) line emission; it has the broadest line width, FWHM $=860 \mathrm{~km} \mathrm{~s}^{-1}$, among the CO-detected $z \sim 6$ quasars (Wang et al. 2007, 2010). We observed the line at the redshift of $z=5.8441 \pm 0.0013$ derived from the high-order $\mathrm{CO}$ detections and found no clear detection in a velocity-averaged map averaging over $1070 \mathrm{~km} \mathrm{~s}^{-1}$ made at the full resolution of $1^{\prime \prime} .09 \times 0.76$. At a lower resolution of 2 "' $19 \times 1$.'.96, marginal signal $(2.8 \sigma)$ appears on the map (Figure 3), with a double-peaked morphology along the east-west direction. The optical quasar position is 0.8 away 

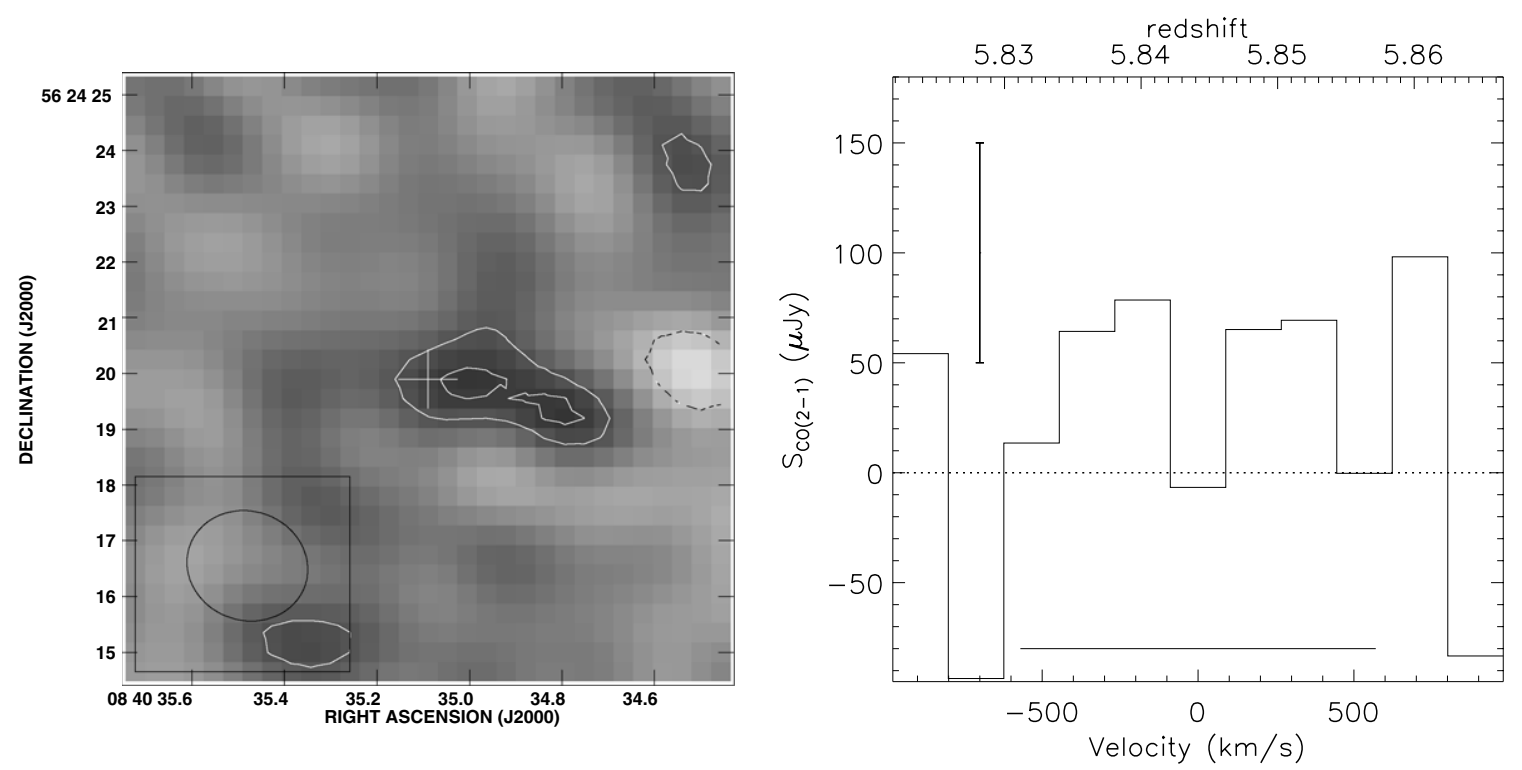

Figure 3. Left: velocity-averaged map of the $\mathrm{CO}(2-1)$ line emission from J0840+5624 at a resolution of 2 !. $19 \times 1$ 1.'96, averaged over -535 to $535 \mathrm{~km} \mathrm{~s}{ }^{-1}$. The contour levels are $(-2,2,3) \times 18 \mu \mathrm{Jy}_{\text {beam }}^{-1}$, and the $1 \sigma$ on the map is $21 \mu \mathrm{Jy}_{\text {beam }}{ }^{-1}$. The cross denotes the optical position of the quasar. Right: spectrum at the position of the east peak, binned to a channel width of $20 \mathrm{MHz}\left(178 \mathrm{~km} \mathrm{~s}^{-1}\right)$. The zero velocity corresponds to a redshift of $z=5.8441$ measured from the high-order CO emission (Wang et al. 2010). The error bar denotes the typical $\pm 1 \sigma \mathrm{rms}$ value. The dotted line shows the zero intensity level and the solid line at the bottom denotes $v_{\text {FWZI }}$ of the high- $J$ CO lines (Wang et al. 2010).

from the east peak. We plot the spectrum at the position of the east peak in the right panel of Figure 3, and there is only very marginal signal $(1 \sigma-2 \sigma)$ over $\sim-500$ to $500 \mathrm{~km} \mathrm{~s}^{-1}$, i.e., the typical velocity range of the $\mathrm{CO}(6-5)$ and (5-4) line emission (Wang et al. 2010). The CO (2-1) line flux estimated with the surface brightness of the east peak is $0.062 \pm 0.022 \mathrm{Jy} \mathrm{km} \mathrm{s}^{-1}$ (Table 1). However, the signal is indeed marginal, and deeper observations with a wider bandwidth are required to improve the measurement.

J0210-0456. This object is the highest redshift quasar known to date with $z=6.438 \pm 0.004$ determined from the object's Mg II $\lambda 2798$ line emission (Willott et al. 2010b). We searched for CO (2-1) line emission in the $128 \mathrm{MHz}$ window centered at the $\mathrm{Mg}$ II redshift but did not detect it. Here we assume a line width of $800 \mathrm{~km} \mathrm{~s}^{-1}$, which is the typical full width at zero intensity $\left(v_{\mathrm{FWZI}}\right)$ value found with samples of high- $z$ CO-detected quasars (Coppin et al. 2008; Wang et al. 2010) to estimate the upper limit of the line intensity. The $1 \sigma \mathrm{rms}$ noise level on the map averaged over this velocity range is $\sigma_{\mathrm{rms}}=16 \mu \mathrm{Jy}_{\mathrm{beam}}{ }^{-1}$, and the $3 \sigma$ upper limit of the line flux is estimated as $3 \sigma_{\text {rms }} \mathrm{V}_{\mathrm{FWZI}}=0.038 \mathrm{Jy} \mathrm{km} \mathrm{s}^{-1}$. The corresponding $3 \sigma$ upper limit of the line luminosity is $L^{\prime} \mathrm{CO}(2-1)<1.28 \times 10^{10} \mathrm{~K} \mathrm{~km} \mathrm{~s}^{-1} \mathrm{pc}^{2}$ (see Equation (3) in Solomon \& Vanden Bout 2005). However, we cannot rule out that the $\mathrm{Mg}$ II line emission is significantly offset from the quasar host galaxy redshift and $\mathrm{CO}(2-1)$ line falls outside the $128 \mathrm{MHz}$ window. The continuum emission is also undetected with the other window centered at $32.1 \mathrm{GHz}$, and the channel-averaged map yields a $3 \sigma$ upper limit of $<54 \mu \mathrm{Jy}$.

$J 1623+3112$. This object is detected in [C II] $158 \mu \mathrm{m}$ fine structure line emission by F. Bertoldi et al. (2011, in preparation), but undetected in $250 \mathrm{GHz}$ dust continuum (Wang et al. 2007). We searched for the CO (2-1) line in the $128 \mathrm{MHz}$ bandwidth window centered at the $[\mathrm{C}$ II] redshift of $z=$ $6.2605 \pm 0.0005$ and did not detect the line. The rms on the map averaged over a velocity range of $800 \mathrm{~km} \mathrm{~s}^{-1}$ is $26 \mu \mathrm{Jy} \mathrm{beam}^{-1}$. This yields a $3 \sigma$ upper limit of $<0.062 \mathrm{Jy} \mathrm{km} \mathrm{s}^{-1}$ for the line flux and $<2.0 \times 10^{10} \mathrm{~K} \mathrm{~km} \mathrm{~s}^{-1} \mathrm{pc}^{2}$ for the line luminosity. The
$3 \sigma$ upper limit of the continuum emission at $35 \mathrm{GHz}$ measured with $\mathrm{A} / \mathrm{C}$ IFs is $<75 \mu \mathrm{Jy}$.

\section{DISCUSSION}

We have observed molecular CO (2-1) line emission toward five quasars at $z \sim 6$ using the EVLA, and detections/marginal detection have been obtained from the three objects that have the strong FIR dust continuum emission. This is consistent with the picture of massive star formation fueling by a large amount of molecular gas in these young quasar hosts. The detection of [C II] in $\mathrm{J} 1623+3112$ is also likely to be a sign of star formation, but the current sensitivity of our EVLA observations cannot detect molecular CO from the host galaxy. J0927+2001 and $\mathrm{J} 0840+5624$ were previously detected strongly in the CO (6-5) and (5-4) transitions. CO (2-1) line emission has been detected and marginally resolved in the host galaxy of J0927+2001 over a scale of $\sim 10 \mathrm{kpc}$. The molecular gas masses $\left(M_{\text {gas }}\right)$ estimated from the $\mathrm{CO}(2-1)$ line peak surface brightness and the total intensity on the velocity-averaged map are listed in Table 1, assuming a CO luminosity-to-gas mass conversion factor of $\alpha=0.8 M_{\odot}\left(\mathrm{K} \mathrm{km} \mathrm{s}^{-1} \mathrm{pc}^{2}\right)^{-1}$ appropriate for local ultraluminous infrared galaxies (Solomon et al. 1997; Downes et al. 1998). These estimates are 1.7 and 2.5 times higher than the value of $(1.8 \pm 0.3) \times 10^{10} M_{\odot}$ estimated from the highorder CO transitions (Carilli et al. 2007; Wang et al. 2010). We plot the CO excitation ladder of this source in Figure 4, together with the results of large velocity gradient (LVG) modeling of the highly excited molecular gas components (gas densities of order $10^{4} \mathrm{~cm}^{-3}$, kinetic temperatures of $50-60 \mathrm{~K}$, and peak at $J \geqslant 6$ ) found in other high- $z$ FIR and CO luminous quasars and nearby starburst galaxies (Riechers et al. 2006, 2009; Güsten et al. 2006). We normalize the models to the high-order CO transitions. The CO (2-1) line flux measured with the peak surface brightness on the velocity-averaged map is consistent/ marginally consistent with the values expected by these singlecomponent models, while the total line flux integrated over the line-emitting area falls above all the models. This may 


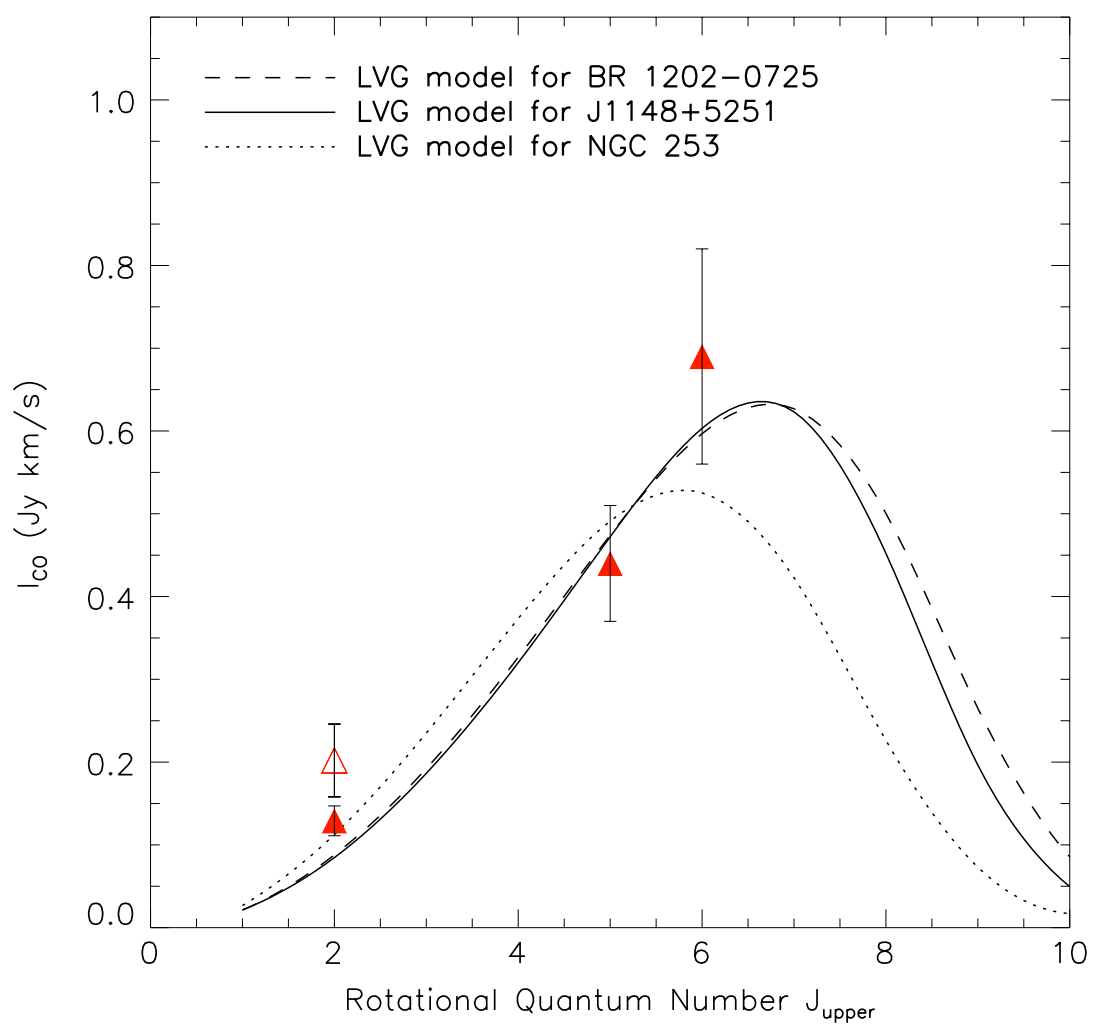

Figure 4. CO excitation ladder of J0927+2001. The filled triangle at $J=2$ is the CO (2-1) line flux measured with the peak surface brightness on the velocity-averaged map, while the open triangle is derived with the total intensity integrated over the line-emitting area of $2.7 \times 2$." 4 . The error bars show the $1 \sigma$ uncertainties of the line fluxes. The solid and dashed lines show the LVG models of $\mathrm{J} 1148+5251$ with kinetic temperature, $T_{\mathrm{kin}}=50 \mathrm{~K}$, molecular hydrogen density, $\rho_{\mathrm{gas}}\left(\mathrm{H}_{2}\right)=10^{4.2} \mathrm{~cm}^{-3}$, from Riechers et al. (2009) and BR 1202-0725 with $T_{\text {kin }}=60 \mathrm{~K}, \rho_{\mathrm{gas}}\left(\mathrm{H}_{2}\right)=10^{4.1} \mathrm{~cm}^{-3}$, from Riechers et al. (2006), respectively. The dotted line represents the LVG model of the central region of the nearby starburst galaxy NGC 253 with $T_{\text {kin }}=60 \mathrm{~K}, \rho_{\text {gas }}\left(\mathrm{H}_{2}\right)=10^{3.9} \mathrm{~cm}^{-3}$ from Güsten et al. (2006). These models are fitted to the $\mathrm{CO}(6-5)$ and (5-4) transitions. We have not made any correction for the continuum emission at the $\mathrm{CO}(2-1)$ line frequency as there is no significant detection. The continuum contamination to the $\mathrm{CO}(2-1)$ line flux is estimated to be $\leqslant 5 \%$ based on the FIR dust continuum determined with previous (sub)mm detections (Wang et al. 2010).

(A color version of this figure is available in the online journal.)

suggest the existence of additional low excitation gas in the central $\sim 10 \mathrm{kpc}$ region as was found in the submillimeter galaxy AzTEC-3 at $z=5.3$ (Riechers et al. 2010) and the nearby starburst galaxy M82 (Weiß et al. 2005). However, there are still large uncertainties in the measurements of all three transitions, and observations of other $\mathrm{CO}$ transitions are necessary to address whether there are multiple $\mathrm{CO}$ excitation components in the quasar host galaxy. Our observations show no evidence of excess CO (2-1) line emission and an additional low excitation component in the host galaxy of $\mathrm{J} 0840+5624$.

The $\mathrm{C}$ array imaging of the $\mathrm{CO}(2-1)$ line emission from $\mathrm{J} 1429+5447$ has resolved the molecular gas into two distinct peaks with a spatial separation of $\sim 6.9 \mathrm{kpc}$; the quasar position is consistent with the west peak. There is no clear velocity offset $\left(26 \pm 60 \mathrm{~km} \mathrm{~s}^{-1}\right)$ between the two components. These results suggest a gas-rich, major merging system with two distinct components that are comparable in CO luminosity and molecular gas mass. The west component of this system is in a radio-loud quasar phase. Similar quasar-starburst systems with multiple $\mathrm{CO}$ emission peaks were previously found in the $\mathrm{CO}$ luminous quasars BRI 1202-0725 at $z=4.7$ (Omont et al. 1996; Carilli et al. 2002), BRI 1335-0417 at $z=4.4$ (Riechers et al. 2008), and J1148+5251 at $z=6.42$ (Walter et al. 2004). These systems demonstrate the early phase of quasar-galaxy formation in which both active galactic nuclei and starburst activities are triggered by major mergers and the molecular gas in the nuclear region is not fully coalesced (Narayanan et al. 2008). We will expect further high-resolution observations with the EVLA in $\mathrm{C}$ or $\mathrm{B}$ array to constrain the gas surface density and dynamics, and with ALMA or the Plateau de Bure Interferometer to resolve the dust continuum and distributed star formation in these young quasar host galaxies.

This work is based on observations carried out with the Expanded Very Large Array (NRAO). The National Radio Astronomy Observatory (NRAO) is a facility of the National Science Foundation operated under cooperative agreement by Associated Universities, Inc. We acknowledge support from the Max-Planck Society and the Alexander von Humboldt Foundation through the Max-Planck-Forschungspreis 2005. Dominik A. Riechers acknowledges support from NASA through Hubble Fellowship grant HST-HF-51235.01 awarded by the Space Telescope Science Institute, which is operated by the Association of Universities for Research in Astronomy, Inc., for NASA, under contract NAS 5-26555. Michael A. Strauss acknowledges the support of NSF grant Ast-0707266.

Facilities: EVLA

\section{REFERENCES}

Becker, R. H., White, R. L., \& Helfand, D. J. 1995, ApJ, 450, 559 Bertoldi, F., Carilli, C. L., Cox, P., et al. 2003a, A\&A, 406, L55 Bertoldi, F., Cox, P., Neri, R., et al. 2003b, A\&A, 409, L47 Carilli, C. L., Kohno, K., Kawabe, R., et al. 2002, AJ, 123, 1838 Carilli, C. L., Neri, R., Wang, R., et al. 2007, ApJ, 666, L9 
Coppin, K. E. K., Swinbank, A. M., Neri, R., et al. 2008, MNRAS, 389, 45 Downes, D., \& Solomon, P. M. 1998, ApJ, 507, 615

Fan, X., Hennawi, J. F., Richards, G. T., et al. 2004, AJ, 128, 515

Fan, X., Strauss, M. A., Richards, G. T., et al. 2006, AJ, 131, 1203

Frey, S., Paragi, Z., Gurvits, L. I., Gabányi, K. É., \& Cseh, D. 2011, in Proc. 10th European VLBI Network Symp. (10th EVN Symp.), 023

Greve, T. R., Bertoldi, F., Smail, I., et al. 2005, MNRAS, 359, 1165

Güsten, R., Philipp, S. D., Weiß, A., \& Klein, B. 2006, A\&A, 454, L115

Ivison, R. J., Papadopoulos, P. P., Smail, I., et al. 2011, MNRAS, 412, 1913

Ivison, R. J., Smail, I., Papadopoulos, P. P., et al. 2010, MNRAS, 404, 198

Kovács, A., Chapman, S. C., Dowell, C. D., et al. 2006, ApJ, 650, 592

Maiolino, R., Cox, P., Caselli, P., et al. 2005, A\&A, 440, L51

Narayanan, D., Li, Y., Cox, T. J., et al. 2008, ApJS, 174, 13

Omont, A., Petitjean, P., Guilloteau, S., et al. 1996, Nature, 382, 428

Perley, R. A., Chandler, C. J., Butler, B. J., \& Wrobel, J. M. 2011, ApJ, 739, L1

Petric, A. O., Carilli, C. L., Bertoldi, F., et al. 2003, AJ, 126, 15

Priddey, R. S., Isaak, K. G., McMahon, R. G., Roboson, E. I., \& Pearson, C. P. 2003, MNRAS, 344, L74

Riechers, D. A., Capak, P. L., Carilli, C. L., et al. 2010, ApJ, 720, L131

Riechers, D. A., Walter, F., Bertoldi, F., et al. 2009, ApJ, 703, 1338
Riechers, D. A., Walter, F., Carilli, C. L., Bertoldi, F., \& Momjian, E. 2008, ApJ, 686, L9

Riechers, D. A., Walter, F., Carilli, C. L., et al. 2006, ApJ, 650, 604

Robson, I., Priddey, R. S., Isaak, K. G., \& McMahon, R. G. 2004, MNRAS, 351, L29

Scott, S. E., Fox, M. J., Dunlop, J. S., et al. 2002, MNRAS, 331, 817

Solomon, P. M., Downes, D., Radford, S. J. E., \& Barrett, J. W. 1997, ApJ, 478, 144

Solomon, P. M., \& Vanden Bout, P. A. 2005, ARA\&A, 43, 677

Spergel, D. N., Bean, R., Doré, O., et al. 2007, ApJS, 170, 377

Wagg, J, Wang, R., Carilli, C., et al. 2008, GBT Memo 256

Walter, F., Bertoldi, F., Carilli, C., et al. 2003, Nature, 424, 406

Walter, F., Carilli, C. L., Bertoldi, F., et al. 2004, ApJ, 615, L17

Walter, F., Riechers, D., Cox, P., et al. 2009, Nature, 457, 699

Wang, R., Carilli, C. L., Beelen, A., et al. 2007, AJ, 134, 617

Wang, R., Carilli, C. L., Neri, R., et al. 2010, ApJ, 714, 699

Wang, R., Carilli, C. L., Wagg, J., et al. 2008, ApJ, 687, 848

Weiß, A., Walter, F., \& Scoville, N. Z. 2005, A\&A, 438, 533

Willott, C. J., Albert, L., Arzoumanian, D., et al. 2010b, AJ, 140, 546

Willott, C. J., Delorme, P., Reylé, C., et al. 2010a, AJ, 139, 906 